\title{
Highlights of CP 2000
}

\author{
John Ellis ${ }^{\mathrm{a}}$

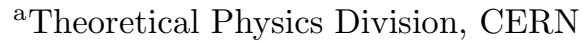 \\ CH 1211 Geneva 23
}

\begin{abstract}
Various developing topics in $\mathrm{CP}$ violation are reviewed. There are many theoretical reasons to hope that the CKM paradigm may be incomplete. It is surely too soon to be claiming new physics in $\epsilon^{\prime} / \epsilon$ or in $D^{0}-\bar{D}^{0}$ mixing, but rare $K$ decays offer interesting places to search for new physics. It is probably also premature to see a clash between global CKM fits and current estimates of $\sin \beta$ and $\gamma$, where much more precise data will soon be available. There are interesting possibilities to look for CP violation in neutrino oscillations and in Higgs physics. Rapid progress can be expected now that CP violation is moving to the top of the particle physics agenda.
\end{abstract}

CERN-TH/2000-297 hep-ph/0011396

\section{Preface}

My personal interest in $\mathrm{CP}$ violation is driven by the search for physics beyond the Standard Model, and this bias guides the following remarks. You all know that the Standard Model is sadly deficient. It is theoretically very unsatisfactory, despite agreeing with all confirmed accelerator data. The Standard Model does not explain the particle quantum numbers (Q, I, Y, Colour) and contains 19 arbitrary parameters. Three of these are its independent gauge couplings, and there is a CP-violating strong interaction vacuum angle $\theta_{Q C D}$, to which we return later. The arbitrary Yukawa couplings are linked to the 6 quark masses, 3 charged-lepton masses, 3 weak mixing angles and the CP-violating Kobayashi-Maskawa phase. Finally, there are the $W^{ \pm}$and Higgs boson masses: is the latter about $115 \mathrm{GeV}$ [1]?

The first clear evidence for physics beyond the Standard Model has come from neutrino oscillations [2], which are most plausibly interpreted in terms of 3 non-zero neutrino masses and 3 neutrino mixing angles. In addition, there are expected to be 3 CP-violating neutrino phases, one of which is observable in scattering experiments, with the other two playing a potential rôle in neutrinoless double- $\beta$ decay. There may well be additional parameters associated with neutrino mass generation, e.g., for more Higgs fields and/or heavy singlet neutrinos.

Additional parameters beyond the Standard Model appear when one considers gravity and cosmology, such as Newton's constant, the cosmological 'constant' and at least one parameter to characterize inflation. Moreover, at least one additional CP-violating parameter is needed to explain the baryon asymmetry of the Universe, which cannot be explained by any of the parameters mentioned above [3].

As already mentioned, we know that some physics beyond the Standard Model is needed to explain neutrino oscillations and, we hope, link them to other physics. The Higgs boson might be one doorway to new physics: if it weighs 115 $\mathrm{GeV}$, there must be some new physics at an energy $\lesssim 10^{6} \mathrm{GeV}$ [4], to prevent the Higgs potential from becoming unstable at this scale [5]. To my 
mind, the default option for this new physics is supersymmetry, as discussed shortly.

What are the prospects for observable physics beyond the Standard Model in CP-violating quantities, such as $\epsilon^{\prime} / \epsilon$ [6]円, $D^{0}-\bar{D}^{0}$ mixing [9] or $\sin 2 \beta[10$ 12]? As discussed later, it is really too soon to interpret any of these as evidence for new physics. On the other hand, there are some intriguing prospects for finding physics beyond the standard Model in rare $K$ and/or $B$ decays.

As emphasized here by Masiero [13], supersymmetry is a framework for physics beyond the Standard Model that is well motivated by the naturalness (or hierarchy) problem of electroweak symmetry breaking. This suggests that sparticles weigh $\lesssim 1 \mathrm{TeV}$, making supersymmetry a testable hypothesis that is already tightly constrained by experiments, in particular on $\mathrm{CP}$ violation [14]. To simplify, let us assume $R$ conservation. Then, if the soft supersymmetry-breaking scalar masses $m_{0}$ and gaugino masses $m_{1 / 2}$ are assumed to be universal, and also the trilinear $A$ terms, there are 5 real parameters and 2 new $\mathrm{CP}$-violating phases. These may be taken as the phases of $A$ and $m_{1 / 2}$, in this 'minimal' supersymmetric model (often called the CMSSM or mSUGRA). In this case, the CP-violating asymmetry in $b \rightarrow s \gamma$ decay may be a promising signature. If one relaxes the universality assumption, there are 60 real parameters and $43 \mathrm{CP}$-violating phases. If one makes the 'reasonable' assumption that all the universality-breaking parameters are of the same order as the corresponding quark and lepton masses: $\Delta \tilde{m}=\mathcal{O}\left(m_{q}\right)$, then there may also be supersymmetric signatures in $\epsilon_{K}, \epsilon^{\prime} / \epsilon$ and $K_{L}^{0} \rightarrow \pi^{0} e^{+} e^{-}$. One may even ask the question whether all the observed $\mathrm{CP}$ violation, i.e., both $\epsilon_{K}$ and $\epsilon^{\prime} / \epsilon$, could originate from supersymmetry 15. Finally, one might be 'hopeful' and assume no close connection between flavour breaking for conventional and supersymmetric particles. In this case, there could be many experimen-

${ }^{1}$ For the first non-zero estimate of $\epsilon^{\prime} / \epsilon$ in the Standard Model, see 17. Paradoxically, the confirmation that $\epsilon^{\prime} / \epsilon \neq$ 0 has killed one candidate for new physics, namely the superweak model of CP violation in the $K^{0}$ system [8]. tal signatures, including most notably $d_{n}, \mu \rightarrow e \gamma$ and $B \rightarrow K_{S}(\phi$ or $\pi)$.

\section{Light Quarks: $u, d, s$}

As we heard here from Hinds [16], the experimental upper limit on the neutron electric dipole moment is $d_{n}<6.3 \times 10^{-26}$ e.cm. This is far above the Standard Model prediction $d_{n} \sim$ $10^{-31} \mathrm{e} . \mathrm{cm}$, providing an extensive opportunity to search for new physics. In particular, supersymmetric models often predict large values of $d_{n}$, which has often been quoted as constraining new supersymmetric phases $\phi$ to the percent level. However, there are many possibilities for cancellations among different diagrams, e.g., charginos vs gluinos [17] and/or diagrams involving $d$ and $s$ quarks [18]. The latter are present in the neutron wave function at the $10 \%$ level, and often have supersymmetric amplitudes that are larger by a factor $m_{s} / m_{d} \sim 20$. Thus one should be cautious before inferring very strong constraints on supersymmetric phases. It should also not be forgotten that $d_{n}$ is uniquely sensitive to $\theta_{Q C D}$, constraining it to be $\lesssim 10^{-9}$ : a subject to which we return later.

The electric dipole moments of other systems are also interesting. For example, that of ${ }^{199} \mathrm{Hg}$ is sensitive to $\mathrm{CP}$-violating nucleon-nucleon interactions, though these are difficult to calculate reliably. The dipole moment of the electron is also interesting, though there may still be cancellations between symmetric diagrams. The electric dipole moment of the muon is expected in many models to be larger than that of the electron by a factor $m_{\mu} / m_{e} \sim 200$, and might provide an interesting opportunity for the BNL $(g-2)_{\mu}$ ring, or for future muon storage rings.

The pioneering CP-violating observable $\epsilon_{K}$ is difficult to calculate reliably in the Standard Model. Certainly, its measured value is qualitatively consistent with the CKM prediction, but it is subject to uncertainties in non-perturbative hadronic matrix elements. Therefore, to my mind it is better not to use $\epsilon_{K}$ in global fits, and I would 
be difficult to convince myself of any signature for new physics in $\epsilon_{K}$.

As already mentioned, it is ironic that the confirmation of a non-zero value for $\epsilon^{\prime} / \epsilon$ killed one possible example of physics beyond the Standard Model, namely the superweak theory [8]. However, any conclusions from the value of $\epsilon^{\prime} / \epsilon$ may be premature before its experimental value has settled down further. Once again, it is predicted qualitatively correctly by the Standard Model. However, $\epsilon^{\prime} / \epsilon$ is particularly difficult to calculate exactly, because of large cancellations between contributions associated with different nonperturbative matrix elements. For this reason, it will also be quite hard to convince oneself of any need for new physics in $\epsilon^{\prime} / \epsilon$, until one has a better handle on these hadronic matrix elements 2 . They have been tackled using a variety of phenomenological approaches, including the chiral quark model [20] and the $1 / N_{C}$ expansion [21]. The best answers may eventually be supplied by lattice calculations [22], but these are not yet very precise.

Rare $K$ decays [23 provide the best lightquark opportunity to look for new physics. The Standard Model calculations are relatively reliable, and far below the present experimental upper limits 3 . New physics might appear in a flavour-changing $Z$ vertex, $Z_{s d}$, in an anomalous dipole moment for $\gamma$ or gluon emission, $\frac{1}{M} \bar{q} \sigma^{\mu \nu} f_{\mu \nu} q$, or in some four-fermion operator, $\frac{1}{M^{2}}(\bar{f} f)(\bar{f} f)[13,25$. The best prospects may be offered by $Z_{s d}$, which is unsuppressed by a potentially large mass $M$. In contrast to $K^{+} \rightarrow \pi^{+} \bar{\nu} \nu$, the Standard Model predictions for the rare decay $K_{L}^{0} \rightarrow \pi^{0} \bar{\nu} \nu$ and the direct contribution to the $K_{L}^{0} \rightarrow \pi^{0} e^{+} e^{-}$amplitude are well below the experimental measurement limits. Somewhat larger values are possible in supersymmetric models with minimal flavour violation, i.e., the same pattern as in the Standard Model, and considerably larger values in a more general class of supersymmetric models with arbitrary flavour violation, or

\footnotetext{
${ }^{2}$ For a recent discussion of the importance of final-state $\pi \pi$ interactions, see [19].

${ }^{3}$ Except for $K^{+} \rightarrow \pi^{+} \bar{\nu} \nu$ 24.
}

with a general parametrization of the $Z_{s d}$ vertex, constrained by $\epsilon_{K}, \epsilon^{\prime} / \epsilon$ and $K_{L}^{0} \rightarrow \mu^{+} \mu^{-}$[13].

There is an active outgoing programme on $K^{+} \rightarrow \pi^{+} \bar{\nu} \nu$ decay [26], led currently by the BNL E787 $\rightarrow$ E949 experiment, aiming at a sensitivity of 5 to 10 events within the Standard Model. There is also a proposal at FNAL to gather $\sim 100 K^{0} \rightarrow \pi^{0} \bar{\nu} \nu$ decays, and proposals at both BNL and FNAL to gather up to $\sim 100 K_{L}^{0} \rightarrow \pi^{0} \bar{\nu} \nu$ decays. In view of the interest of these decays for flavour physics in general, and $\mathrm{CP}$ violation in particular, I hope the resources will be made available to conduct such next-generation rare $K$ decay experiments, particularly if one recalls the amount of resources directed towards $B$ physics.

\section{Medium-Heavy Quark: $c$}

One expects $D^{0}-\bar{D}^{0}$ mixing to be very small in the Standard Model: $x \equiv \Delta m / r \lesssim 0.001$ and $y \equiv \Delta \Gamma / \Gamma$ smaller still, providing another opportunity for new physics to strut its stuff. There is currently some excitement [27] generated by the FOCUS report of a $2-\sigma$ signal for $y \sim 0.03$ [9]. This excitement is probably premature, since the effect is not confirmed. It is important to clarify what is the maximum possible value in the Standard Model, which may be rather larger than some estimates, through presumably not as large as the central value of FOCUS. There is also the issue of compatibility between the FOCUS result and that of CLEO [28], which would be easier if there is a large strong-interaction phase. Large CP violation in the $D^{0}-\bar{D}^{0}$ system would be a particularly strong indication for physics beyond the Standard Model.

\section{Global CKM fits}

Before discussing the search for CP violation in $B$ meson decays, let us review the Standard Model predictions provided by global fits of the CKM parameters [29]. Several convergent mea- 


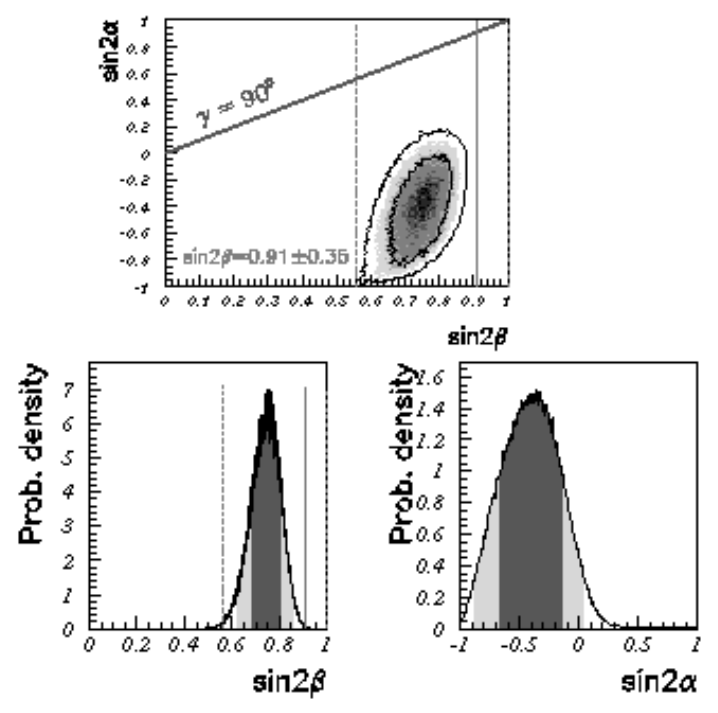

Figure 1. Results of a global CKM fit, displaying preferred ranges of $\sin 2 \beta$ and $\alpha$, and indicating that $\gamma<90^{\circ}$ 29.

surements indicate that

$\bar{\rho}=0.206 \pm 0.043$,

$\bar{\eta}=0.339 \pm 0.044$

including the $B^{0}-\bar{B}^{0}$ mass difference $\Delta m_{d}$, the lower limit on the $B_{s}^{0}-\bar{B}_{s}^{0}$ mass difference $\Delta m_{s}$ [30], and the ratio $\left|V_{u b}\right| /\left|V_{c b}\right|$. The ranges (11) are supported by the experimental value of $\epsilon_{K}$, though the theoretical uncertainties in its interpretation are rather greater. As seen in Fig. 1, the corresponding prediction for $\beta\left(=\phi_{1}\right)$ is:

$\sin 2 \beta=0.723 \pm 0.069$

and it would be quite surprising if experiments find $\sin 2 \beta<0.5$, as discussed later. On the other hand, the value of $\alpha$ is rather more uncertain:

$\sin 2 \alpha=-0.28 \pm 0.27$
The global Standard Model fit predicts quite confidently that $\gamma$ is in the first quadrant:

$\gamma=58.5 \pm 6.9^{0}, \quad<90^{0} @ 99.8 \%$ c.l.

This result is mainly enforced by the $\Delta m_{s}$ constraint, and it is worth remembering that a joint analysis of the existing experiments yields a 2.5 - $\sigma$ effect corresponding to $\Delta m_{s} \sim 18 / p s$ [30], as expected in the Standard Model on the basis of the other CKM measurements.

\section{Heavy Quark: $b$}

With the advent of the $\mathrm{B}$ factories, a new age is dawning for $\mathrm{CP}$ violation studies. However, there is still some early-morning fog!

$\sin 2 \beta$

The present experimental measurements:

$$
\begin{aligned}
\sin 2 \beta= & 0.79 \pm 0.39 \pm 0.16 \\
& 0.12 \pm 0.37 \pm 0.09 \\
& 0.45_{-0.44-0.09}^{+0.43+0.07}
\end{aligned}
$$

are mutually compatible within their large errors, and may be combined to yield

$\sin 2 \beta=0.42 \pm 0.24$

This value is also compatible at the $1-\sigma$ level with the global CKM fit prediction (2). However, this has not prevented theorists from speculating on the possible consequences if it eventually transpires that $\sin 2 \beta<0.5$ [31].

Could we have misunderstood the Standard Model so badly that $\sin 2 \beta<0.5$ is possible within the conventional CKM framework? This would require reducing the estimated value of $\left|V_{u b} / V_{c b}\right|$ by about $30 \%$, or increasing the estimate of $\xi \equiv \sqrt{\frac{B_{B_{s}}}{B_{B_{d}}}} \frac{f_{B_{s}}}{f_{B_{d}}}$ to $\gtrsim 1.4$, or increasing $\hat{B}_{K}$ to $\gtrsim 1.3$, or some combination of these effects. Among these, a different value of $\left|V_{u b} / V_{c b}\right|$ may be the least implausible [32]. 
Alternatively, there might be new physics in $B^{0}-\bar{B}^{0}$ mixing, which could yield $\sin 2 \beta<0.5$ if the new physics has a non-trivial phase: $2 \beta \rightarrow$ $2\left(\beta+\theta_{d}\right)$, or if it changes the magnitudes of $\Delta m_{d, s}$ by factors $\Gamma_{d, s}: 0.5 \lesssim \Gamma_{d} \lesssim 1$ and $\Gamma_{s} / \Gamma_{d} \gtrsim 1.1$. Another possibility is new physics in $K^{0}-\bar{K}^{0}$ mixing, which would alter the interpretation of $\epsilon^{\prime}$ [32].

However, I emphasize again that there is no need for new physics to explain the value of $\sin 2 \beta$ (yet). We await with impatience the next round of analyses!

$\underline{\sin 2 \alpha}$

The prospects for measuring this quantity in $B_{0} \rightarrow \pi^{+} \pi^{-}$decay may now be less problematic than in 1999, for two reasons. One is that the two new measurements of the branching ratio:

$$
\begin{aligned}
B\left(B_{0} \rightarrow\right. & \left.\pi^{+} \pi^{-}\right)= \\
& \left\{\begin{array}{l}
\left(9.3_{(-2.3-1.4}^{+2.6+1.2)}\right) \times 10^{-6} \\
(6.3 \pm 43]
\end{array}\right.
\end{aligned}
$$

raise the possibility that this decay mode may be less suppressed relative to $K^{+} \pi^{-}$than was suggested by the previous CLEO data [28]. This would have the twin advantages of making it easier to extract from the background and of reducing the prospective penguin pollution. The second ray of hope for $\sin 2 \alpha$ is that the systematic perturbative QCD factorization approach [35], based on transition form factors with calculable corrections, should permit better calculations of penguin pollution from first principles [36.

Recall that the CP-violating asymmetry in $\stackrel{(-)}{B} \rightarrow \pi^{+} \pi^{-}$decay can be written as

$$
A_{C P}(t)=-S \cdot \sin \left(\Delta m_{d} t\right)+C \cdot \cos \left(\Delta m_{d} t\right)
$$

where $S=\sin 2 \alpha$ and $C=0$ in the absence of penguins. In the presence of calculable penguins, a tight correlation between the value of $\alpha$ and $S$ can be predicted, and the prediction for $S$ checked by verifying the corresponding prediction for the

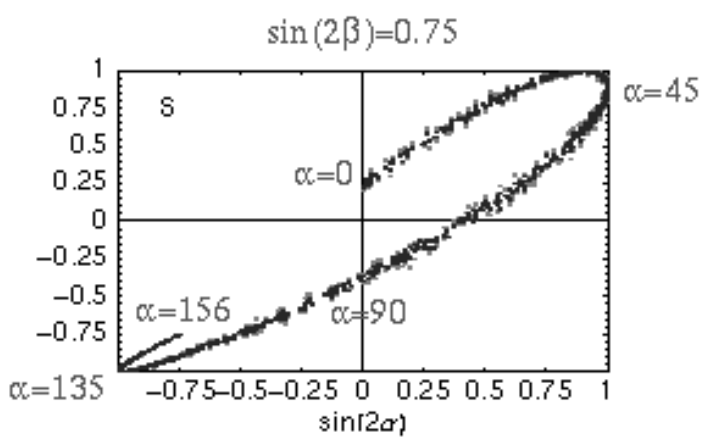

Figure 2. The correlation between $S$ and $\alpha$ expected in the $Q C D$ factorization approach to penguins [30].

direct $\mathrm{CP}$-violating quantity $C$ [36], as seen in Fig. 2.

$\underline{\gamma}$ There has recently been intense theoretical exploration of the bounds on $\gamma$ that can be set indirectly by measurements of ratios of branching ratios for $B \rightarrow K \pi$ and $\pi \pi$ decays [37. In particular, there has been some excited comment that perhaps $\gamma>90^{\circ}$, based on the early (and imprecise) measurements of these branching ratios. If true, this might be an interesting hint of physics beyond the Standard Model, which strongly prefers $\gamma<90^{\circ}$ as discussed earlier. However, any talk of a $\gamma$ 'crisis' is premature, in view of the various sources of theoretical uncertainty (renormalization scale, quark masses, wave functions, ...) and the large experimental errors (so far). In particular, what will happen to the ratio of $\pi^{+} \pi^{-} / K^{+} \pi^{-}$? Let us wait for an experimental consensus to emerge.

\section{Very Heavy Quark: $t$}

The production and decays of $\bar{t} t$ pairs will be an interesting place to look for $\mathrm{CP}$ violation in the future 38], but was not discussed at this meeting, so I do not discuss it further here. 

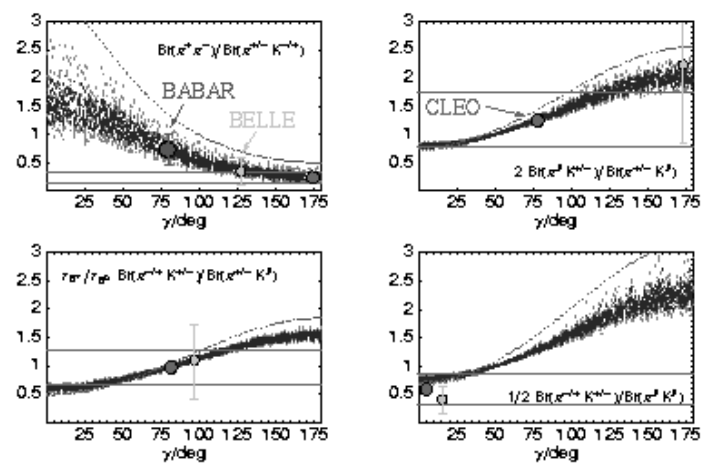

Figure 3. Measurements of exclusive $B$ decays by CLEO, BaBar and Belle do not yet permit any conclusion whether $\gamma<$ or $>90^{\circ}$ [3]].

\section{Neutrino Masses and Oscillations}

There is no good reason why $m_{\nu}=0$, since vanishing masses are generally associated with exact gauge symmetries (e.g., the photon and the $U(1)$ gauge invariance of QED), and there is no massless gauge boson coupling to lepton number. A neutrino mass term $m_{\nu} \nu \cdot \nu$ is possible if there is a $\Delta L=2$ interaction, and is a generic feature of GUTs. However, it is even possible in the Standard Model, without new particles or gauge interactions, if one simply introduces a non-renormalizable interaction [39]:

$\frac{1}{M}(\nu H) \cdot(\nu H) \Rightarrow m_{\nu}=\frac{<0|H| 0>^{2}}{M}$

scaled by some new heavy mass scale $M \gg m_{W}$ so that $m_{\nu} \ll m_{q, \ell}$. In GUTs, one normally encounters 'right-handed' singlet neutrinos $\nu_{R}$, and a seesaw mass matrix of the generic form

$\left(\nu_{L}, \nu_{R}\right) \quad\left(\begin{array}{cc}0 & m \\ m^{T} & M\end{array}\right) \quad\left(\begin{array}{c}\nu_{L} \\ \nu_{R}\end{array}\right)$ where the off-diagonal entry $m=\mathcal{O}\left(m_{q, \ell}\right)$, yielding

$m_{\nu}=m \frac{1}{M} m^{T}$

after diagonalization.

The quantities $m, M$ in (10), (11) should be understood as $3 \times 3$ matrices, that cannot in general be expected to be diagonalizable in the same basis as the charged-lepton masses $m_{\ell}$, leading to a neutrino mixing matrix à la CKM [40]:

$V_{M N S}=V_{L} V_{\nu}^{\dagger}$

with an observable CKM-like phase $\delta$. However, in addition, there are also relative phases in the diagonalized light Majorana neutrino mass matrix $\operatorname{diag}\left(e^{i \alpha}, e^{i \beta}, 1\right)$. These are unobservable at energies $E \ll m_{\nu_{i}}$, but enter into the interpretation of double- $\beta$ decay experiments.

The mixing angles $\theta_{i j}$ and the CP-violating phase $\delta$ appearing in (12) are observable via neutrino oscillations, and there are indications of non-zero values [2]. In view of the different origin (10,11) for neutrino masses, it should perhaps not be surprising if neutrino mixing angles are very different from the CKM angles. Indeed, atmospheric neutrino experiments favour large mixing between $\nu_{\mu}$ and $\nu_{\tau}$ [41], and solar neutrino experiments favour (less strongly) large mixing between $\nu_{e}$ and some combination of $\nu_{\mu}$ and $\nu_{\tau}$ [42]. On the other hand, atmospheric and reactor neutrino experiments have so far only established upper limits on $\nu_{\mu} \rightarrow \nu_{e}$ mixing.

The next stage of neutrino mixing studies will be led by long-baseline accelerator beam experiments [43], which should measure $\nu_{\mu}$ mixing more precisely, set better limits on (or measure) $\nu_{\mu} \rightarrow \nu_{e}$ mixing, and observe $\tau$ production by $\nu_{\mu} \rightarrow \nu_{\tau}$ oscillations.

The following stage may be led by neutrino factories - muon storage rings that produce intense neutrino beams with known fluxes, spectra 
and charge/flavour separation 444. Such neutrino factories consist of an intense proton source, typically providing several MW of protons at some energy between 2 and $20 \mathrm{GeV}$, a high-power target producing pions, a system to capture, cool and accelerate the muons produced by pion decays, and then a 'ring' to store the muons while they decay. The ring need not be circular, indeed it should have long straight sections (that need not be parallel) producing $\nu$ beams directed towards detectors at different distances.

Such a neutrino factory would further refine measurements of neutrino mixing angles, but the prime interest would be to look for $\mathrm{CP}$ violation 44] via the asymmetry

$A_{C P} \equiv \frac{P\left(\nu_{\mu} \rightarrow \nu_{e}\right)-P\left(\bar{\nu}_{\mu} \rightarrow \bar{\nu}_{e}\right)}{P\left(\nu_{\mu} \rightarrow \nu_{e}\right)+P\left(\bar{\nu}_{\mu} \rightarrow \bar{\nu}_{e}\right)}$

which has the following approximate theoretical expression:

$A_{C P} \simeq 2 c_{13} s_{13} s_{23} \sin \delta \frac{\Delta m_{12}^{2} L}{2 E_{\nu}} \sin ^{2} \frac{\Delta m_{23}^{2} L}{4 E_{\nu}}$

where $\Delta m_{12}^{2}$ is the smaller neutrino mass-squared difference measured in solar neutrino experiments. The latest Super-Kamiokande results favour relatively large $\Delta m_{12}^{2} \gtrsim 2 \times 10^{-5} \mathrm{eV}^{2}$ and a large mixing angle, favouring the measurement of $A_{C P}$.

The measurement of this quantity must contend with the $\mathrm{CP}$-asymmetric matter environment of the Earth. The best significance for measuring $A_{C P}$ is likely to be obtained with a baseline $L \sim 3000 \mathrm{~km}$ 44, 45, e.g., CERN to Northern Scandinavia or the Canary Islands. As seen in Fig. A, there would be good sensitivity to the CP-violating phase $\delta$ with $\gtrsim 10^{21} \mu$ decays. Alternatively, it has been suggested that one could measure $\delta$ with a low-energy $\nu_{\mu}$ beam produced directly ty $\pi$ decay [46], but this seems very difficult with the beam insensitivites currently discussed, and suffers from non-trivial backgrounds 44].

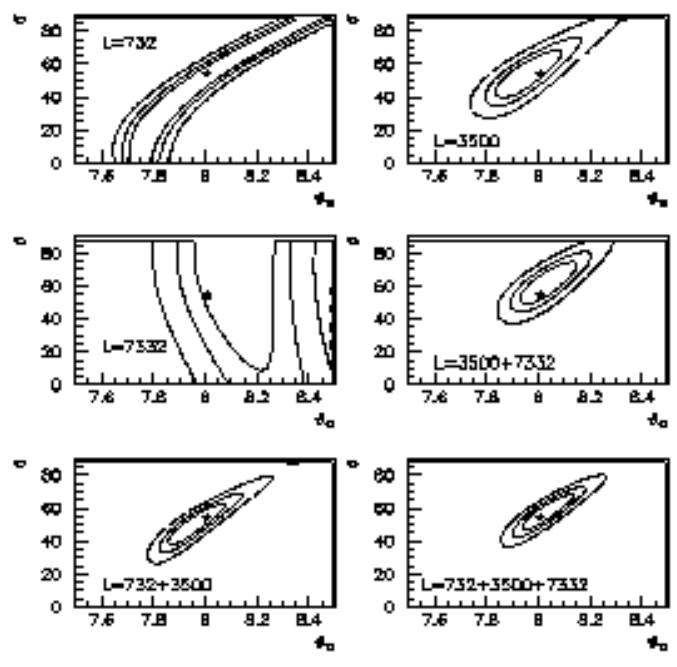

Figure 4. Illustration how neutrino oscillation data over different baselines may be used to measure $\theta_{13}$ and the $C P$-violating phase $\delta$, assuming $10^{2} 1 \mu$ decays and a realistic detector [4].

\section{Higgs Bosons}

$\mathrm{CP}$ violation in the Higgs sector has long been a favoured alternative (or supplement) to the CKM model. The minimal Standard Model cannot accommodate $\mathrm{CP}$ violation in the Higgs sector. In the MSSM, the Higgs sector does not violate CP at the tree level, but may acquire $\mathrm{CP}$ violation via radiative corrections 447. Assuming universal scalar masses $m_{0}$, gaugino masses $m_{1 / 2}$ and trilinear supersymmetry-breaking parameters A, there are two potentially-important sources of CP violation in the MSSM, namely $\operatorname{Arg}\left(A_{t, b}\right)$ and $\operatorname{Arg}\left(m_{\tilde{g}}\right)$. These induce Higgs scalar-pseudoscalar mixing at the one- and two-loop level, respectively 48]:

$\delta M_{S P}^{2} \sim \frac{m_{t}^{4}}{v^{2}} \frac{\mu \operatorname{Im} A_{t}}{32 \pi^{2} m_{\text {susy }}^{2}}+\ldots$ 
In the presence of $\mathrm{CP}$ violation, it is best to parametrize the MSSM Higgs sector in terms of $m_{H^{+}}$and $\tan \beta$, since the pseudoscalar Higgs mass $m_{A}$, the conventional mass parameter choice, is no longer an eigenvalue of the neutral Higgs mass matrix 44].

We have proposed a new MSSM Higgs benchmark scenario (CPX) with maximal $\mathrm{CP}$ violation [49]:

$$
\begin{aligned}
m_{\tilde{q}} & =m_{\tilde{t}}=m_{\tilde{b}} \equiv m_{\text {susy }}, \quad \mu=4 m_{\text {susy }}, \\
\left|A_{t}\right| & =\left|A_{b}\right|=2 m_{\text {susy }}, \quad\left|m_{\tilde{g}}\right|=1 \mathrm{TeV} \\
\operatorname{Arg}\left(A_{t}\right) & =\operatorname{Arg}\left(m_{\tilde{g}}\right)=90^{9}
\end{aligned}
$$

This we contrast with a CP-conserving scenario (MAX) with maximal mixing, in which

$A_{t}=A_{b}=\sqrt{6} m_{\text {susy }}$,

$\mu=m_{\tilde{B}}=m_{\tilde{W}}=200 \mathrm{GeV}$

$\operatorname{Arg}\left(A_{t}\right)=\operatorname{Arg}\left(m_{\tilde{g}}\right)=0$

The LEP physics reaches for the CPX and MAX scenarios are compared in Fig. 5 4 49 .

Among the intriguing possibilities offered by the CP-violating MSSM is level crossing when the two lightest neutral Higgses have $m_{H_{1}} \simeq m_{H_{2}}$. As seen in Fig. 6, this is accompanied by a stronglysuppressed ZZ coupling:

$\left.\left.g_{H_{1} Z Z}\right|_{C P X} \ll g_{H_{1} Z Z}\right|_{S M}$

resulting in suppressed production of the lightest MSSM Higgs at LEP 2. On the other hand,

$\left.\left.g_{H_{1} \bar{b} b}\right|_{C P X} \simeq g_{H_{1} \bar{b} b}\right|_{M A X}$

and the dominant decay modes is expected to be $\bar{b} b$, as usual. In view of (18), we find that an $H_{1}$ as light as $50 \mathrm{GeV}$ might have escaped detection at LEP 49.

An intriguing feature of the level-crossing scenario, also seen in Fig. 6, is the fact that the
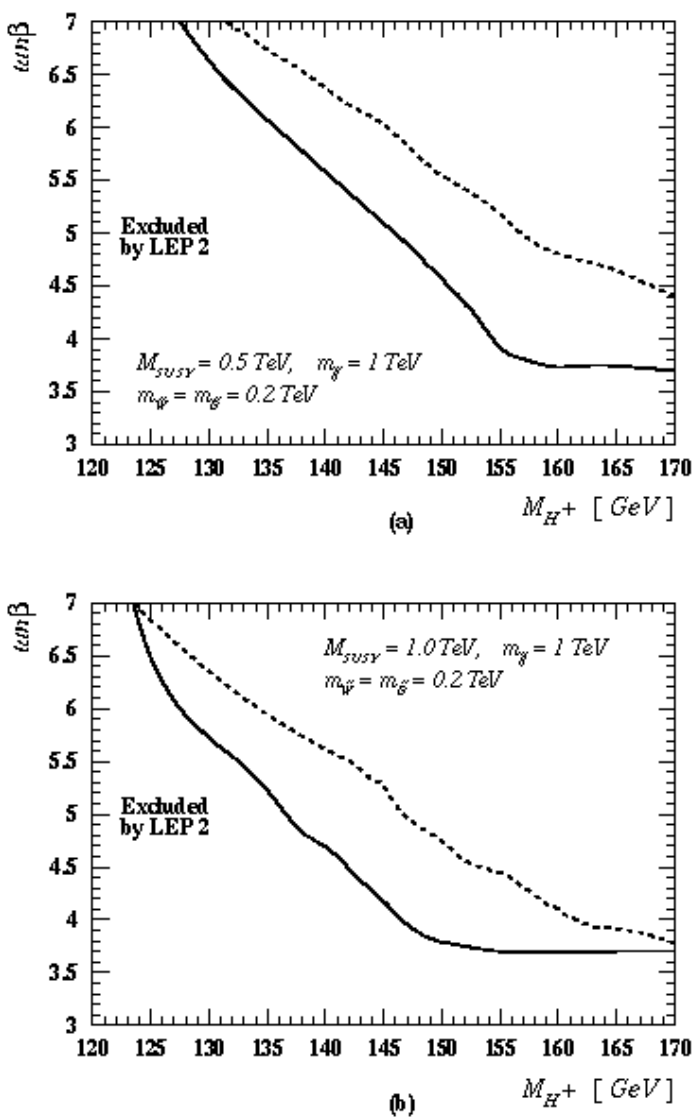

Figure 5. Comparison between the regions of the $\left(m_{H^{+}}, \tan \beta\right)$ plane allowed in the maximal $C P_{-}$ violating scenario (10) and the CP-conserving scenario (17), for two different supersymmetrybreaking scales 49 . 


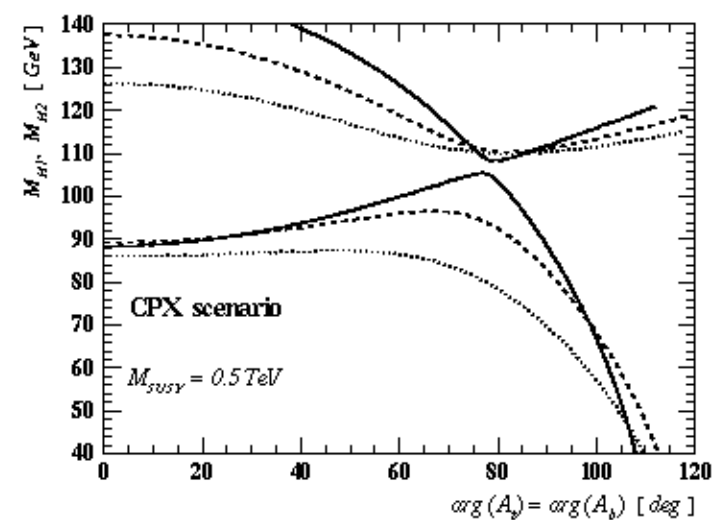

(a)

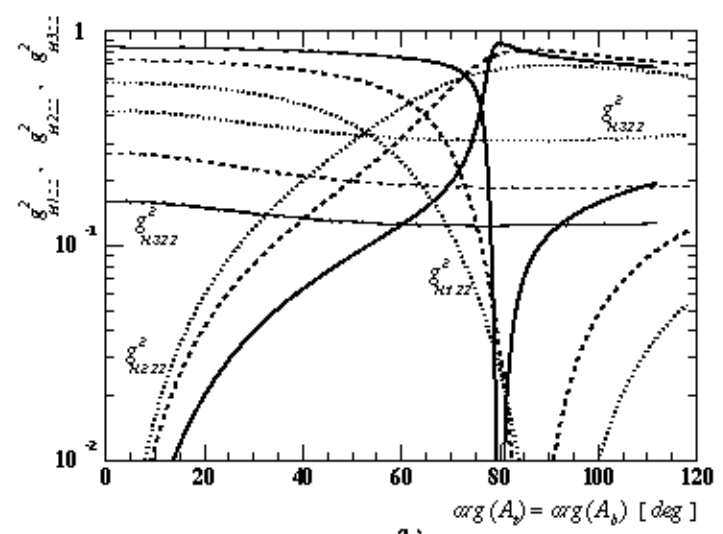

(b)

Figure 6. Variation of the two lightest neutral Higgs masses $M_{H 1, H 2}$ and the $H i-Z-Z$ couplings, as functions of the CP-violating angle $\operatorname{Arg}\left(A_{t, b}\right)$. Note the level crossing in the top panel and the vanishing $H 1-Z-Z$ coupling in the lower panel 49 . second Higgs $\mathrm{H}_{2}$ weighs $\sim 115 \mathrm{GeV}$. If the current weak Higgs 'signal' at LEP [1] turns out to be confirmed, is it conceivable that the experiments are seeing the second MSSM Higgs boson? It is also not excluded experimentally that there might exist another Higgs weighing $\sim 95 \mathrm{GeV}$ with $\left.g_{H Z Z}^{2} \simeq 0.1 g_{H Z Z}^{2}\right|_{S M}$, which could be accommodated in the MSSM if $m_{H^{+}} \sim 160 \mathrm{GeV}$, $\tan \beta \sim, \operatorname{Arg}\left(A_{t}\right) \sim 85^{\circ} 49$.

It would require an extensive programme of measurements at the LHC, an $e^{+} e^{-}$linear collider and a muon collider to unravel potential $\mathrm{CP}$ violation in the MSSM Higgs sector.

\section{Baryogenesis}

As already emphasized, the fact that we exist is evidence for physics beyond the Standard Model. Generating a cosmological baryon asymmetry requires a departure from thermal equilibrium as well as sufficient $\mathrm{CP}$ violation [50,3]. In the Standard Model, the LEP lower limit on $m_{H}$ implies that the electroweak phase transition is not first order [51], and, in addition, the Standard Model has too little $\mathrm{CP}$ violation. The following are some of the favoured scenarios for baryogenesis.

\section{GUT Boson Decay?}

This scenario might seem remote from experimental test, but many such scenarios would induce a renormalization of the non-perturbative QCD vacuum parameter $\theta_{Q C D}$, and hence a neutron electric dipole moment that is close to the present experimental upper limit [52]:

$d_{n} \gtrsim 10^{-27}$ e.cm

to be contrasted with the Standard Model value $d_{n} \sim 10^{-31} \mathrm{e} . \mathrm{cm}$. Of course, the interpretation of any observation of $d_{n}$ in the range (20) would be ambiguous, but at least it offers some prospect of testing GUT baryogenesis scenarios.

Heavy Neutrino Decay?

A variation on the GUT boson decay scenario is the out-of-equilibrium decay of a heavy 'right- 
handed' neutrino seen in (10) [53]. In this case, the relevant $\mathrm{CP}$ violation would be associated with the Dirac neutrino couplings appearing in the off-diagonal entries in (10) $\lambda=m /<$ $0|H| 0>$. These generate a cosmological lepton asymmetry, which is then partially converted by electroweak sphalerons into a baryon asymmetry. Could this scenario be tested by measuring CP violation in $\nu$ oscillations? Unfortunately, they measure a different form of $\mathrm{CP}$ violation, although there might well be some relation between them.

Supersymmetric Electroweak Transitions? In the MSSM, if there is a light stop $\tilde{t}$, as well as a relatively light Higgs boson, it is possible for the electroweak phase transition to be first order [51,54]. Moreover, as we have seen, large CP violation in the MSSM cannot be excluded. Possible tests of this scenario include finding the Higgs and/or a light $\tilde{t}$, as well as supersymmetric $\mathrm{CP}$ violation. This scenario is the only one to offer a prospect of relating the observed value of $n_{B} / n_{\gamma}$ directly to quantities measurable in the laboratory.

\section{0. (In-)Conclusions}

The experimental situation with regard to $\mathrm{CP}$ violation is evolving rapidly: after $\epsilon_{K}$ and the confirmation that $\epsilon^{\prime} / \epsilon \neq 0$, we can look forward soon to measurements of $\sin 2 \beta, \sin 2 \alpha, \gamma$ and other $\mathrm{CP}$-violating observables in $\mathrm{B}$ decays. There are also prospects for observing $\mathrm{CP}$ violation elsewhere, e.g., in electric dipole moments. In parallel, theoretical tools such as the heavyquark effective theory and factorization are also developing rapidly. It is too soon to get excited about new physics in $\epsilon^{\prime} / \epsilon, D^{0}-\bar{D}^{0}$ mixing, sin $2 \beta$ or $\gamma$, but we should be vigilant. $\mathrm{CP}$ violation is moving from the qualitative era to a quantitative one, when deviations from the Standard Model may well appear. In the longer run, new prospects for probing $\mathrm{CP}$ violation are opening up, e.g., in the neutrino sector and perhaps even the Higgs sector.
Generally, the flavour problem is now moving to the top of the particle physics agenda, with the completion of precision electroweak measurements at LEP and possibly the first element in the solution of the mass problem with a hint of the Higgs boson 11. Our excitement makes it difficult to wait for the next conference in this series, but, while we do, there are grounds for worry - that the CKM model may be right - and also for hope - that it may be wrong, or at least incomplete.

\section{REFERENCES}

1. ALEPH collaboration,

http://alephwww.cern.ch/ and preprint CERN-EP/2000-138, hep-ex/0011045;

DELPHI collaboration,

http://delphi.web.cern.ch/Delphi/;

L3 collaboration, http://13www.cern.ch/

and preprint CERN-EP/2000-140, hepex/0011043;

OPAL collaboration, http://opal.web.cern.ch/Opal/ PPwelcome.html and Physics Note PN468, http://opal.web.cern.ch/Opal/pubs/ physnote/html/pn468.html.

2. For a review and references, see: J. Ellis, Summary talk at 19th International Conference On Neutrino Physics And Astrophysics, June 2000, Sudbury: hep-ph/0008334.

3. A. Riotto and M. Trodden, Ann. Rev. Nucl. Part. Sci. 49 (1999) 35; A. Riotto, talk at this meeting.

4. J. Ellis, G. Ganis, D.V. Nanopoulos and K.A. Olive, hep-ph/0009355.

5. G. Altarelli and G. Isidori, Phys. Lett. B337 (1994) 141;

6. A. Alavi-Harati et al., KTeV Collaboration, Phys. Rev. Lett. 83 (1999) 22; R. Kessler, for the $\mathrm{KTeV}$ Collaboration, talk at this meeting; V. Fanti et al., NA48 Collaboration, Phys. Lett. B465 (1999) 335; I. Mikulec, for the NA48 Collaboration, talk at this meeting.

7. J. Ellis, M. K. Gaillard and D. V. Nanopoulos, Nucl. Phys. B109 (1976) 213.

8. L. Wolfenstein, Phys. Rev. Lett. 13 (1964) 562. 
9. S. Bianco, for the FOCUS Collaboration, talk at this meeting.

10. K. Ackerstaff et al., OPAL Collaboration, Eur. Phys. J. C5 (1998) 379; F. Abe et al., CDF Collaboration, Phys. Rev. Lett. 81 (1998) 5513; A. Cerri, for the CDF and D0 Collaborations, talk at this meeting and FERMILAB-CONF-00-272-E (2000); R. Barate et al., ALEPH Collaboration, Phys. Lett. B492 (2000) 259.

11. D. G. Hitlin, for the BABAR Collaboration, hep-ex/0011024; G. Sciolla, for the BaBar Collaboration, talk at this meeting.

12. H. Aihara, Belle Collaboration, hepex/0010008; Y. Sakai, for the Belle Collaboration, talk at this meeting.

13. D. A. Demir, A. Masiero and O. Vives, Phys. Lett. B479 (2000) 230; A. Masiero and O. Vives, hep-ph/0007320; A. Masiero, talk at this meeting.

14. See, for example: G. L. Kane, hep$\mathrm{ph} / 0008190$.

15. See, for example: A. Masiero and H. Murayama, Phys. Rev. Lett. 83 (1999) 907; M. Brhlik, L. Everett, G. L. Kane, S. F. King and O. Lebedev, Phys. Rev. Lett. 84 (2000) 3041.

16. J. M. Pendlebury and E. A. Hinds, Nucl. Instrum. Meth. A440 (2000) 471; E.A. Hinds, talk at this meeting.

17. M. Brhlik, G. J. Good and G. L. Kane, Phys. Rev. D59 (1999) 115004.

18. J. Ellis and R. A. Flores, Phys. Lett. B377 (1996) 83; A. Bartl, T. Gajdosik, W. Porod, P. Stockinger and H. Stremnitzer, Phys. Rev. D60 (1999) 073003.

19. E. Pallante and A. Pich, Phys. Rev. Lett. 84 (2000) 2568.

20. M. Fabbrichesi, talk at this meeting and hep$\mathrm{ph} / 0009321$.

21. T. Hambye, G. O. Kohler, E. A. Paschos and P. H. Soldan, Nucl. Phys. B564 (2000) 391; J. Bijnens and J. Prades, hep-ph/0009155.

22. A. J. Buras, M. Ciuchini, E. Franco, G. Isidori, G. Martinelli and L. Silvestrini, Phys. Lett. B480 (2000) 80; . Ciuchini and G. Martinelli, hep-ph/0006056; M. Ciuchini, talk at this meeting; A. Soni, talk at this meeting.

23. For a recent review, see: A.R. Barker and S.H. Kettell, hep-ex/0009024.

24. S. Adler et al., E787 Collaboration, Phys. Rev. Lett. 84 (2000) 3768.

25. A. J. Buras, P. Gambino, M. Gorbahn, S. Jäger and L. Silvestrini, hep-ph/0007313; L. Silvestrini, talk at this meeting;

26. L. Littenberg, T. Yamanaka and P.S. Cooper, talks at this meeting.

27. S. Bergmann, Y. Grossman, Z. Ligeti, Y. Nir and A. A. Petrov, hep-ph/0005181.

28. D. E. Jaffe, talk at this meeting and hepex/0011021, and references therein.

29. F. Caravaglios, F. Parodi, P. Roudeau and A. Stocchi, hep-ph/0002171; F. Parodi, talk at this meeting.

30. LEP/SLD/CDF Working Group on B oscillations, http://lepbosc.web.cern.ch/LEPBOSC/.

31. A. L. Kagan and M. Neubert, Phys. Lett. B492 (2000) 115; J. P. Silva and L. Wolfenstein, hep-ph/0008004; G. Eyal, Y. Nir and G. Perez, JHEP 0008 (2000) 028; Z. Xing, hep-ph/0008018; A. J. Buras and R. Buras, hep-ph/0008273.

32. For a recent review, see: Y. Nir, hep$\mathrm{ph} / 0008226$.

33. BaBar Collaboration, hep-ex/0008057; M.-H. Schune, for the Babar Collaboration, talk at this meeting.

34. K. Trabelsi, for the Belle Collaboration, talk at this meeting;

35. M. Beneke, G. Buchalla, M. Neubert and C. T. Sachrajda, Phys. Rev. Lett. 83 (1999) 1914 and Nucl. Phys. B591 (2000) 313.

36. M. Neubert, talk at this meeting and hep$\mathrm{ph} / 0011064$.

37. M. Neubert and J. L. Rosner, Phys. Lett. B441 (1998) 403 and Phys. Rev. Lett. 81 (1998) 5076; A. J. Buras and R. Fleischer, Eur. Phys. J. C11 (1999) 93 and hepph/0008298; G. W. S. Hou, hep-ph/0009197; X.-G. He, Y.-K. Hsiao, J.-Q. Shi, Y.-L. Wu and Y.-F. Zhou, hep-ph/0011337; among earlier work, see for example: R. Fleischer, Phys. Lett. B365 (1996) 399.

38. See, for example: D. Atwood, S. Bar-Shalom, 
G. Eilam and A. Soni, hep-ph/0006032.

39. R. Barbieri, J. Ellis and M. K. Gaillard, Phys. Lett. B90 (1980) 249.

40. Z. Maki, M. Nakagawa and S. Sakata, Prog. Theor. Phys. 28 (1962) 870.

41. S. Fukuda et al., Super-Kamiokande Collaboration, Phys. Rev. Lett. 85 (2000) 3999 and references therein.

42. Y. Suzuki, talk at 19 th International Conference On Neutrino Physics And Astrophysics, June 2000, Sudbury: http://nu2000.sno.laurentian.ca/ Y.Suzuki/index.html.

43. K. Nishikawa, talk at 30th Int. Conf. On High-Energy Physics Osaka, July/August 2000 ,

http://ichep2000.hep.sci.osaka-u.ac.jp/ scan/0801/pl/nishikawa/index.html.

44. J.J. Gomez-Cadenas, talk at this meeting.

45. A. De Rujula, talk at 19th International Conference On Neutrino Physics And Astrophysics, June 2000, Sudbury:

http://nu2000.sno.laurentian.ca/ A.DeRujula/index.html.

46. J. Sato, talk at Nufact00, Monterey, May 2000, hep-ph/0008056; B. Richter, hep$\mathrm{ph} / 0008222$.

47. See, for example: A. Pilaftsis and C. E. Wagner, Nucl. Phys. B553 (1999) 3.

48. M. Carena, J. Ellis, A. Pilaftsis and C. E. Wagner, Nucl. Phys. B586 (2000) 92.

49. M. Carena, J. Ellis, A. Pilaftsis and C. E. Wagner, hep-ph/0009212.

50. A. D. Sakharov, Pisma Zh. Eksp. Teor. Fiz. 5 (1967) 32.

51. See, for example, M. Laine, talk at Int. Workshop on Strong and Electroweak Matter, June 2000, Marseille, hep-ph/0010275.

52. J. Ellis, M. K. Gaillard, D. V. Nanopoulos and S. Rudaz, Phys. Lett. B99 (1981) 101 and Nature 293 (1981) 41.

53. M. Fukugita and T. Yanagida, Phys. Lett. B174 (1986) 45.

54. M. Carena, J. M. Moreno, M. Quiros, M. Seco and C. E. Wagner, hep-ph/0011055. 